\title{
A Seleção de Áreas para Conservação na Mata Atlântica Brasileira: Revisão dos Estudos Voltados para Priorização Espacial
}

\author{
Rosana Cristina Pezzi D’Arrigo ${ }^{1}$, Maria Lucia Lorini ${ }^{2} \&$ Henrique Rajão $^{3}$
}

Recebido em 21/10/2019 - Aceito em 22/06/2020 1 Instituto Chico Mendes de Conservação da Biodiversidade. Equipe da Base do Rio de Janeiro da Gerência Regional 04-sudeste, Rio de
Janeiro/RJ, Brasil. CEP: 22.461-000. < rosacris142019@outlook.com>.
2 Universidade Federal do Estado do Rio de Janeiro, Instituto de Biociências, Departamento de Ciências Naturais. Rio de Janeiro/RJ, Brasil.
CEP: 22.290-255. <mluc.lorini@gmail.com>.
3 Pontifícia Universidade Católica do Rio de Janeiro/PUC-Rio, Centro de Ciências Biológicas e da Saúde, Departamento de Biologia, Rio de
Janeiro/RJ, Brasil. CEP: 22.451-900. < henrique.rajao@gmail.com>.

RESUMO - A Mata Atlântica (MA) possui um histórico ininterrupto de degradação ambiental, o mais antigo e contínuo do Brasil. Por outro lado, foi nesse bioma que ocorreram as primeiras demarcações de áreas protegidas no Brasil. A MA é também muito estudada no âmbito da biologia da conservação. As metodologias para a avaliação, seleção e priorização de áreas para alocação de estratégias de conservação, no contexto do Planejamento Sistemático para Conservação (PSC), vêm sendo desenvolvidas a partir do ano 2000. Realizou-se aqui a revisão bibliográfica da atividade de pesquisa relacionada ao PSC e à aplicação de métodos de priorização espacial para a conservação na MA Brasileira. Nessa busca, foram levantados vinte e um estudos acadêmicos e dois não acadêmicos. Observa-se uma tendência nesses estudos, principalmente os acadêmicos, de utilizarem grupos taxonômicos específicos (por exemplo, espécies de vertebrados terrestres) como indicadores de biodiversidade. A baixa reversão dos estudos que utilizam o PSC e os métodos para priorização espacial para a conservação na MA Brasileira em ações de conservação é observada também na maior parte dos estudos que adotam essas metodologias.

Palauras-chave: Áreas prioritárias; conservação da biodiversidade; seleção de reservas; unidade de conservação.

\section{The Selection of Conservation Areas in the Brazilian Atlantic Forest: Review of Studies for Spatial Prioritization}

ABSTRACT - The Atlantic Forest (AF) has a history of uninterrupted environmental degradation which is the oldest and most continuous in Brazil. On the other hand, it was the AF biome where the first protected areas were demarcated in Brazil. The AF is broadly studied in the field of Biological Conservation. The methodologies for evaluating, selecting and prioritizing areas for allocating conservation strategies, in the context of the Systematic Planning for Conservation (SPC), have been developed since the year 2000. A bibliographic review of the research activity related to the SPC and the application of spatial prioritization methods for conservation in the Brazilian Atlantic Forest was carried out here. In this research, twenty-one academic and two non-academic studies were surveyed. There is a tendency of the surveyed studies, mainly academic ones, to use specific taxonomic groups as species of terrestrial vertebrates as indicators of biodiversity. Low setback of studies that use the SPC and the methods for spatial prioritization for conservation in Brazilian AF in conservation actions is also observed in most studies that adopt these methodologies.

Keywords: Biodiversity conservation; conservation unit; priority areas; reserve selection.

\section{La Selección de Áreas de Conservación en el Bosque Atlántico Brasileño: Revisión de Estudios para la Priorización Espacial}

RESUMEN - El Bosque Atlántico (BA) tiene una historia ininterrumpida de degradación ambiental, la más antigua y continua de Brasil. Por otro lado, fue en este bioma donde se produjeron las primeras 
demarcaciones de áreas protegidas en Brasil. Se estudia BA también ampliamente en el campo de la biología de la conservación. Se han desarrollado las metodologías para la evaluación, selección y priorización de áreas para la asignación de estrategias de conservación, en el contexto de la Planificación Sistemática para la Conservación (PSC) desde el año 2000. Aquí se realizó una revisión bibliográfica de la actividad de investigación relacionada a la PSC y la aplicación de métodos de priorización espacial para la conservación en el BA Brasileño. En esta investigación, se encuestaron veintiún estudios académicos y dos no académicos. Hay una tendencia de los estudios encuestados, principalmente académicos, a utilizar grupos taxonómicos específicos como especies de vertebrados terrestres como indicadores de biodiversidad. La baja reversión de los estudios que utilizan la PSC y los métodos de priorización espacial para la conservación en el BA Brasileño en acciones de conservación también se observa en la mayoría de los estudios que adoptan estas metodologías.

Palabras clave: Áreas prioritarias; conservación de la biodiversidad; selección de reservas; unidad de conservación.

\section{Introdução}

A Mata Atlântica (MA) é a segunda maior floresta pluvial tropical do continente americano (Oliveira-Filho \& Fontes 2000). Essa floresta abriga 19.355 espécies de plantas, sendo endêmicas desse bioma $40 \%$ das espécies (Forza et al. 2012). Estimativas apontam para a ocorrência de, aproximadamente, 2,2 mil espécies de vertebrados na MA (Paglia \& Pinto 2010). A flora e a fauna da MA podem incluir de $1 \%$ a $8 \%$ do total de espécies do mundo (Silva \& Casteleti 2003).

Por outro lado, o histórico de degradação da MA é antigo e contínuo (Ayres et al. 2005, Tabarelli et al. 2005, Ribeiro et al. 2009). A Mata Atlântica Brasileira (MAB) sofreu séculos de exploração madeireira e de degradação por agricultura, pecuária, silvicultura e caça (Laurence 2009). Esse quadro foi agravado pelo fato de a região abrigar mais de $70 \%$ de toda a população brasileira. Cerca de $80 \%$ do PIB brasileiro é gerado nos estados situados na região da MAB (CEPF 2001, De Marques et al. 2016). O resultado desse longo histórico de perda e fragmentação de habitat é a redução da cobertura florestal da $\mathrm{MAB}$, que se encontra reduzida a, aproximadamente, $11 \%$ a $16 \%$ de sua extensão original, sendo que $80 \%$ dos fragmentos remanescentes são menores que 50ha (Ribeiro et al. 2009). A MA está repleta de espécies ameaçadas de extinção. A título de exemplo, $80 \%$ das 199 espécies de aves endêmicas da região estão atualmente ameaçadas de extinção (Laurence 2009). Portanto, sua riqueza biológica, exposta ao referido histórico de degradação ambiental, coloca a MA entre as áreas mais prioritárias para ações de conservação no mundo, sendo considerada um dos 25 hotspots para a conservação da biosfera (Myers et al. 2000, Mittermeier et al. 2005).
No Brasil, os esforços pioneiros para conservação, na forma de áreas protegidas territorialmente demarcadas, ocorreram na área de abrangência da MA, onde foi instituída a primeira área protegida, territorialmente demarcada no país, representada pelas "Florestas da Tijuca e das Paineiras", em 1861, no Rio de Janeiro (Medeiros 2006). Os primeiros parques nacionais foram igualmente criados em áreas de MA, como o Parque Nacional do Itatiaia, em 1937, e os Parques Nacionais do Iguaçu e da Serra dos Órgãos, em 1939 (Diegues 2001, Cabral 2002, Ferreira 2004). Esses três parques nacionais foram criados no contexto histórico iniciado pela criação do Parque Nacional de Yellowstone, em 1872, sendo este o primeiro parque nacional instituído nos Estados Unidos da América e no mundo (Morsselo 2001, Araújo 2012). As motivações para a criação dos parques nacionais relacionam-se com as noções de bem público nacional de usufruto democrático e com caráter de monumento para a proteção de sítios de grande beleza cênica (Runte 1979).

A ideia de que devam existir áreas protegidas se aplica, portanto, aos primeiros parques nacionais $e$, posteriormente, avança para a criação de sistemas de áreas protegidas, as quais se reproduziram mundialmente até a atualidade (Morsello 2001). A evolução das tipologias de áreas protegidas (Tabela 1) teve origem nos valores recreativos e na proteção de cenários espetaculares, como grandes cânions e cascatas, inicialmente propostos como objetivos dos parques (Runte 1979). Paulatinamente, essas áreas protegidas passaram a ser criadas como locais para a conservação de espécies, habitat e outros objetivos afetos a uma noção científica relacionada à biologia da conservação (Morsello 2001). 
Tabela 1 - Tipologia de Áreas Protegidas associadas ao SNUC e a IUCN (Adaptado de MMA 2007b).

\begin{tabular}{|c|c|c|}
\hline CATEGORIAS IUCN & \multicolumn{2}{|l|}{ CATEGORIAS SNUC } \\
\hline \multirow{2}{*}{$\begin{array}{l}\text { Ia - Reserva de Proteção Integral - área manejada } \\
\text { principalmente para pesquisa }\end{array}$} & RESERVA BIOLÓGICA & \multirow{6}{*}{$\begin{array}{l}\text { PROTEÇÃO } \\
\text { INTEGRAL }\end{array}$} \\
\hline & ESTAÇÃO ECOLÓGICA & \\
\hline $\begin{array}{c}\text { Ib - Área Selvagem - área protegida e manejada } \\
\text { principalmente para proteção da natureza }\end{array}$ & $\begin{array}{l}\text { NÃO HÁ CATEGORIA EQUIVALENTE } \\
\text { NO SNUC }\end{array}$ & \\
\hline $\begin{array}{l}\text { II - Parque Nacional - área protegida e manejada } \\
\text { principalmente para proteção dos ecossistemas e recreação }\end{array}$ & PARQUE NACIONAL & \\
\hline \multirow{2}{*}{$\begin{array}{c}\text { III - Monumento Natural - área protegida e manejada } \\
\text { principalmente para conservação de aspectos naturais } \\
\text { específicos }\end{array}$} & MONUMENTO NATURAL & \\
\hline & REFÚGIO DA VIDA SILVESTRE & \\
\hline \multirow{2}{*}{$\begin{array}{l}\text { IV - Área de Manejo de espécies ou habitat } \\
\text { - área protegida com manejo ambiental }\end{array}$} & $\begin{array}{l}\text { ÁREA DE RELEVANTE INTERESSE } \\
\text { ECOLÓGICO }\end{array}$ & \multirow{5}{*}{$\begin{array}{l}\text { USO } \\
\text { SUSTENTÁVEL }\end{array}$} \\
\hline & $\begin{array}{l}\text { RESERVA PARTICULAR DO } \\
\text { PATRIMÔNIO NATURAL }\end{array}$ & \\
\hline $\begin{array}{c}\text { V - Paisagem Terrestre ou Marinha - área protegida manejada } \\
\text { principalmente para conservação e recreação em paisagens } \\
\text { terrestres e marinhas }\end{array}$ & ÁREA DE PROTEÇÃO AMBIENTAL & \\
\hline \multirow[b]{2}{*}{ VI - Área de manejo de recursos - Área protegida e manejada } & RESERVA EXTRATIVISTA & \\
\hline & $\begin{array}{l}\text { RESERVA DE DESENVOLVIMENTO } \\
\text { SUSTENTÁVEL }\end{array}$ & \\
\hline
\end{tabular}

O Planejamento Sistemático para Conservação (PSC) é um campo da biologia da conservação que tem merecido muita atenção e pesquisa (Pinto \& Bini 2008, Pressey \& Bottrill 2008). O PSC possui em seu escopo uma gama de métodos de priorização espacial para a conservação cujas aplicações atualmente se diversificaram bastante (Margules \& Pressey 2000, Margules \& Sarkar 2007, Moilanen et al. 2009). Originalmente proposto por Margules \& Pressey (2000), é um procedimento para seleção de reservas definidas dentre as tipologias de áreas protegidas das categorias I IV (Tabela 1), conforme o sistema de classificação da União Internacional para a Conservação da Natureza (IUCN 1994) e o Sistema de Unidades de Conservação Brasileiro (SNUC), estabelecido pela Lei $n^{\circ} 9.985$, de 18 de julho de 2000. O SNUC estabelece as normas de criação, ampliação e outras alterações das unidades de conservação brasileiras.

Além disso, o SNUC determina as categorias das unidades de conservação (UCs) brasileiras, dividindo-as em dois grupos: as de proteção integral e as de uso sustentável, cada qual com suas características específicas. O objetivo básico das unidades de proteção integral é preservar a natureza, sendo admitido apenas o uso indireto dos seus recursos naturais, com exceção dos casos previstos em lei. A presença humana, quando permitida, ocorre para visitação ou para realização de pesquisa científica. Já as unidades de uso sustentável visam compatibilizar a conservação da natureza com o uso sustentável dos recursos naturais. Permitem a presença do homem em seu interior, podendo nelas residir.

As categorias de I a IV $e$ as unidades de conservação de proteção integral fazem restrições aos usos diretos e estão associadas à conservação de espécies, habitat e locais com características únicas. Elas foram as primeiras a serem estabelecidas, influenciadas pelo modelo do Parque Nacional de Yellowstone. Associada às restrições de uso direto está uma concepção de preservação do ambiente natural (pristinity) ou ambiente selvagem (wilderness) (Kalamandeen \& Gilson 2006). 
A literatura científica que relaciona a seleção de reservas ao PSC é vasta (Moilanen et al. 2009, Pinto \& Grelle 2009a), sendo que o grande volume de artigos publicados tem sido produzido principalmente nos Estados Unidos e na Austrália (Moilanen et al. 2009, Ribeiro \& Ateu 2019). A revisão dos artigos que abordaram seleção de reservas na América do Sul até 2008, conduzida por Pinto \& Grelle (2009a), encontrou 24 artigos, dos quais somente dois propuseram algum tipo de seleção de reservas na MA. Knight e colaboradores (2009) apontam que a grande maioria dos estudos acadêmicos que aplicam técnicas de priorização espacial não resulta na implementação de ações para conservação. Esses autores observam, no entanto, uma progressiva apropriação dessas técnicas na literatura não acadêmica, pelas agências de governo e organizações não governamentais, para a resolução de problemas explícitos da conservação da biodiversidade. Tal apropriação tem ocorrido de forma isolada da aplicação que se observa na literatura científica (Prendergast et al. 1999, Hopkinson et al. 2000, Pullin et al. 2004).

No presente estudo buscamos traçar um panorama das aplicações de Planejamento Sistemático para Conservação voltado à seleção de áreas para conservação na MAB. Para tanto, realizamos um levantamento dos estudos envolvendo PSC na MAB que foram publicados nos últimos 20 anos, tanto por via acadêmica, em periódicos científicos, como por organizações não governamentais e pelo Governo Brasileiro. A partir dos estudos encontrados, foi feita uma análise cienciométrica sobre os seguintes aspectos: ano de publicação, área de abrangência dos estudos, país de vínculo institucional dos autores, cobertura taxonômica e indicadores (surrogates) de biodiversidade utilizados, objetivos e métodos empregados. Na discussão é feita a contextualização e a revisão desses métodos, $e$ é enfatizada a baixa reversão desses estudos em ações de conservação da biodiversidade na MAB.

\section{Metodologia}

Os resultados deste estudo são baseados em dados compilados por meio de protocolos $\mathrm{de}$ consultas orientadas por palavras-chave em três bases de dados de publicações científicas: Web of Science (WoS) (ISI - Thomson Scientific,
Philadelphia), Scopus e Scielo (Scientific Electronic Library Online). Essas bases de dados são muito utilizadas em análises cienciométricas e em levantamentos bibliográficos (Verbeek et al. 2002, Wilson et al. 2007, Pyšek et al. 2008).

As buscas por artigos se deram por meio da rotina "general search". Foram realizadas buscas com as seguintes palavras-chave: "atlantic forest" AND "conservation biology" OR "biodiversity conservation" OR "systematic conservation planning" OR "spatial conservation prioritization" OR "conservation prioritization" OR "reserve selection" OR "surrogate" OR "nature reserve". Assumiu-se que as buscas utilizando essas palavras-chave capturariam uma parte representativa dos artigos importantes para esta avaliação. Aplicando-se a sintaxe adequada para cada uma das três bases de dados citadas, foram efetuadas buscas para recuperar artigos ou revisões publicadas até dezembro de 2019.

$\mathrm{Na}$ ausência de um protocolo específico para buscar os estudos não acadêmicos que utilizaram métodos de priorização espacial para a conservação na MAB, foi feita uma busca em web pages de organizações não governamentais internacionais e de grande porte que realizam estudos para planejamento de ações de conservação baseados em instrumentos da área de conhecimento da biologia da conservação (para identificar essas instituições, veja Robinson 2006). Da mesma forma, foi consultada a página do Ministério do Meio Ambiente do Brasil (https://www.mma.gov.br/), que é o ponto focal para realização e fomento de estratégias para conservação da biodiversidade relacionadas com a Convenção sobre a Diversidade Biológica (CDB) no Brasil (Marinoni \& Peixoto 2010).

Após um processo de filtragem efetuado com base nos resumos dos estudos recuperados nas buscas, foram retidos para análise apenas aqueles que aplicaram métodos de Planejamento Sistemático para Conservação na Mata Atlântica Brasileira. As seguintes informações foram retiradas dos estudos acadêmicos selecionados: ano de publicação, periódico de publicação do artigo, região em que o estudo foi realizado, objetivos do estudo, tipos de dados utilizados, método e programa utilizados, extensão da área de estudo, nomes dos autores e co-autores e país de vínculo institucional dos autores. As mesmas informações foram levantadas dos estudos não acadêmicos, excluindo-se as informações sobre o periódico da 
publicação e sendo indicada a instituição responsável pelo estudo. Também buscamos em todos os textos indicações que pudessem evidenciar se o estudo teve reversão em implementação de áreas de conservação na MAB.

\section{Resultados}

\section{Estudos acadêmicos}

O resultado das buscas recuperou 7.461 artigos na base Web of Science, 185 na base Scopus e 1.826 na base Scielo. A maior parte desses trabalhos foi eliminada no processo de filtragem, sendo que, no total, excluindo-se as repetições, foram encontrados apenas 21 artigos que aplicaram métodos de priorização espacial para a conservação na MA. Foram identificados 18 artigos (sete exclusivos) na base Scopus, 12 (doze exclusivos) na Web of Science, e nenhum na base Scielo (Tabela 2). Excluindo-se as repetições, esses 21 artigos foram publicados em 14 periódicos: Biodiversity and Conservation, Conservation Biology, Diversity and Distributions, Magistra Cruz das Almas, Forests, Environmental Conservation, Natureza e Conservação, PeerJ, Biological Invasions, Journal for Nature Conservation, Ecological Informatics, Ecology and Evolution, Plos One, Biodiversity Research (Tabela 2).

Tabela 2 - Artigos acadêmicos que aplicaram o Planejamento Sistemático para Conservação da Mata Atlântica.

\begin{tabular}{|c|c|c|c|c|c|c|}
\hline \multirow[b]{2}{*}{ Referência } & \multirow[b]{2}{*}{ Periódico } & \multirow[b]{2}{*}{ Ano } & \multicolumn{3}{|c|}{ Base de dados } & \multirow[b]{2}{*}{ Área de abrangência } \\
\hline & & & Scopus & $\begin{array}{l}\text { Web of } \\
\text { Science }\end{array}$ & Scielo & \\
\hline de Oliveira et al. & Magistra Cruz das Almas & 2019 & & & & Mata Atlântica Brasileira \\
\hline Vasconcelos \& Prado & Journal for Nature Consrvation & 2019 & $\mathrm{x}$ & & & Mata Atlântica e Cerrado \\
\hline Ovando et al. & Journal for Nature Consrvation & 2019 & $\mathrm{x}$ & & & Mata Atlântica Argentina \\
\hline Larossa et al. & Biodiversity and Conservation & 2019 & $\mathrm{X}$ & $\mathrm{X}$ & & Mata Atlântica Brasileira \\
\hline de Lima \& Pinto & Ecological Informatics & 2018 & $\mathrm{X}$ & $\mathrm{X}$ & & Mata Atlântica Brasileira \\
\hline de Oliveira et al. & Biological Invasions & 2018 & $\mathrm{x}$ & $\mathrm{x}$ & & Região Neotropical \\
\hline Vale et al. & $\begin{array}{l}\text { PeerJ - The Journal of Life and } \\
\text { Environmental Scienses }\end{array}$ & 2018 & $\mathrm{X}$ & $\mathrm{X}$ & & Mata Atlântica Brasileira \\
\hline Iacona et al. & PNAS & 2017 & $\mathrm{x}$ & $\mathrm{x}$ & & Mata Atlântica Paraguaia e Austrália \\
\hline Zwiener et al. & Biodiversity Research & 2017 & $\mathrm{x}$ & & & Mata Atlântica Brasileira \\
\hline Crouzeilles et al. & Diversity and Distributions & 2015 & $\mathrm{X}$ & $\mathrm{X}$ & & Mata Atlântica Brasileira \\
\hline Campos et al. & Ecology and Evolution & 2014 & $\mathrm{x}$ & $\mathrm{x}$ & & Mata Atlântica Brasileira \\
\hline Lemes \& Loyola & Plos One & 2013 & $\mathrm{x}$ & & & Mata Atlântica Sul Americana \\
\hline Loyola et al. & Biodiversity and Conservation & 2013 & & $\mathrm{X}$ & & Mata Atlântica Brasileira \\
\hline Izquierdo \& Clark & Forests & 2012 & $\mathrm{x}$ & & & Mata Atlântica Argentina \\
\hline Trindade-Filho et al. & Biodiversity and Conservation & 2012 & $\mathrm{x}$ & $\mathrm{x}$ & & Mata Atlântica Sul Americana \\
\hline Pinto \& Grelle & Environmental Conservation & 2011 & $\mathrm{X}$ & & & Mata Atlântica Brasileira \\
\hline Felinks et al. & Biodiversity and Conservation & 2011 & & $\mathrm{x}$ & & Mata Atlântica Brasileira \\
\hline Grelle et al. & Natureza e Conservação & 2010 & $\mathrm{x}$ & & & Mata Atlântica Brasileira \\
\hline Becker et al. & Diversity and Distributions & 2010 & $\mathrm{X}$ & $\mathrm{X}$ & & Mata Atlântica Brasileira \\
\hline Pinto \& Grelle & Biodiversity and Conservation & $2009 b$ & $\mathrm{x}$ & & & Mata Atlântica Brasileira \\
\hline Loiselle et al. & Conservation Biology & 2003 & $\mathrm{X}$ & $\mathrm{X}$ & & Mata Atlântica Brasileira \\
\hline
\end{tabular}


Apenas 14 artigos aplicaram métodos de Planejamento Sistemático para Conservação na $\mathrm{MAB}$, cujos dados mais importantes são apresentados na Tabela 3. Tratando-se da extensão espacial da área de estudo, 11 desses 14 artigos trataram de toda a MAB (Loiselle et al. 2003; Pinto \& Grelle 2009b, 2011; Loyola et al. 2013; Campos et al. 2014; Crouzeilles et al. 2015; de Lima \& Pinto 2018; Vale et al. 2018; Zwiener et al. 2017; Larossa et al. 2019; de Oliveira et al. 2018), um restringiu-se ao estado do Rio de Janeiro (Grelle et al. 2010) e dois a áreas do estado de São Paulo (Becker et al. 2010, Felinks et al. 2011). $\mathrm{O}$ trabalho de Felinks e colaboradores se restringiu a uma área de $100 \mathrm{~km}^{2}$ de paisagem fragmentada de Mata Atlântica do platô de Ibiúna, localizada $50 \mathrm{~km}$ a oeste de São Paulo.
Em relação à resolução espacial dos estudos, Becker et al. (2010) empregaram dois tipos de unidades de planejamento, de Lima \& Pinto (2018) utilizaram três, e todos os demais estudos utilizaram um único tipo de unidade de planejamento (Tabela 3). Nove artigos utilizaram unidades de planejamento de tamanho constante, quadradas ou hexagonais, em diversas resoluções. A maioria dos estudos empregou células quadradas com resolução espacial entre $1^{\circ}$ e $0,05^{\circ}$. Crouzeilles e colaboradores (2015) utilizaram 71.871 unidades de planejamento de 2.000 ha. Cinco estudos empregaram unidades de planejamento de tamanho irregular, definidas por limites de bacias hidrográficas (Becker et al. 2010), de municípios (Pinto \& Grelle 2011, Larrosa et al. 2018, Vale et al. 2018) ou de fragmentos florestais (Felinks et al. 2011) (Tabela 3).

Tabela 3 - Estudos acadêmicos que aplicaram Planejamento Sistemático para Conservação na Mata Atlântica Brasileira.

\begin{tabular}{|c|c|c|c|c|c|c|c|}
\hline Referência & $\begin{array}{l}\text { País dos } \\
\text { Autores }\end{array}$ & $\begin{array}{l}\text { Dados } \\
\text { (Indicadores) } \\
\text { Utilizados* }\end{array}$ & Objetivos & $\begin{array}{l}\text { Resolução } \\
\text { Espacial } \\
\text { (grão)** }^{*}\end{array}$ & $\begin{array}{l}\text { Extensão } \\
\text { Espacial }\end{array}$ & Método de Seleção & Programa \\
\hline $\begin{array}{l}\text { Loiselle } \\
\text { et al. } 2003\end{array}$ & $\begin{array}{l}\text { EUA, Brasil } \\
\text { e Reino } \\
\text { Unido }\end{array}$ & $\begin{array}{l}\text { Cotingidae } \\
\text { (Aves) }\end{array}$ & $\begin{array}{l}\text { Testar diferentes métodos de } \\
\text { modelagem e suas implicações } \\
\text { nos resultados de seleção de } \\
\text { reservas }\end{array}$ & $\begin{array}{l}\text { Células de } \\
0,25^{\circ}(\sim 27 \mathrm{x} \\
27 \mathrm{~km})\end{array}$ & $\begin{array}{l}\text { Mata Atlântica } \\
\text { Brasileira }\end{array}$ & $\begin{array}{l}\text { Algoritmos sequenciais } \\
\text { baseados em } \\
\text { complementaridade }\end{array}$ & WORLDMAP \\
\hline $\begin{array}{l}\text { Pinto \& } \\
\text { Grelle } \\
2009 b\end{array}$ & Brasil & Primatas & $\begin{array}{l}\text { Verificar a eficiência do sistema } \\
\text { de unidades de conservação de } \\
\text { proteção integral (reservas) para } \\
\text { conservação de primatas }\end{array}$ & $\begin{array}{l}\text { Células de } \\
0,25^{\circ}(\sim 25 \mathrm{x} \\
25 \mathrm{~km})\end{array}$ & $\begin{array}{l}\text { Mata Atlântica } \\
\text { Brasileira }\end{array}$ & $\begin{array}{l}\text { Simulated } \\
\text { annealing usando } \\
\text { complementaridade }\end{array}$ & SITES*** \\
\hline $\begin{array}{l}\text { Grelle et al. } \\
2010\end{array}$ & Brasil & $\begin{array}{l}\text { Tipos de } \\
\text { vegetação }\end{array}$ & $\begin{array}{l}\text { Estabelecer rede de áreas } \\
\text { prioritárias para conservação que } \\
\text { seja representativa do conjunto } \\
\text { das fitofisionomias que ocorrem } \\
\text { no estado }\end{array}$ & $\begin{array}{l}\text { Células de } \\
0,05^{\circ}(\sim 5 \mathrm{X} \\
5 \mathrm{~km})\end{array}$ & $\begin{array}{l}\text { Estado do Rio } \\
\text { de Janeiro }\end{array}$ & $\begin{array}{l}\text { Simulated } \\
\text { annealing usando } \\
\text { complementaridade }\end{array}$ & SITES*** \\
\hline $\begin{array}{l}\text { Becker et al. } \\
2010\end{array}$ & $\begin{array}{l}\text { Brasil e } \\
\text { EUA }\end{array}$ & $\begin{array}{l}\text { Anfíbios } \\
\text { (anuros) }\end{array}$ & $\begin{array}{l}\text { Avaliar a eficiência do sistema } \\
\text { de unidades de conservação } \\
\text { de proteção integral do estado } \\
\text { de São Paulo em relação à } \\
\text { conservação dos anfíbios }\end{array}$ & $\begin{array}{l}\text { 1) Hexágonos } \\
\text { de } 1200 \mathrm{~km}^{2} \\
\text { 2) Bacias } \\
\text { hidrográficas } \\
\text { (tamanho } \\
\text { médio }= \\
11273.9 \mathrm{~km}^{2} \text { ) }\end{array}$ & $\begin{array}{l}\text { Estado de São } \\
\text { Paulo }\end{array}$ & $\begin{array}{l}\text { Simulated } \\
\text { annealing usando } \\
\text { complementaridade }\end{array}$ & MARXAN \\
\hline $\begin{array}{l}\text { Pinto \& } \\
\text { Grelle } 2011\end{array}$ & Brasil & Primatas & $\begin{array}{l}\text { Avaliar a rede de municípios em } \\
\text { relação às áreas de potencial } \\
\text { conflito para conservação de } \\
\text { primatas }\end{array}$ & Municípios & $\begin{array}{l}\text { Estado do Rio } \\
\text { de Janeiro }\end{array}$ & $\begin{array}{l}\text { Simulated } \\
\text { annealing usando } \\
\text { complementaridade }\end{array}$ & MARXAN \\
\hline $\begin{array}{l}\text { Felinks et al. } \\
2011\end{array}$ & $\begin{array}{l}\text { Alemanha } \\
\text { e Brasil }\end{array}$ & $\begin{array}{l}\text { Anfíbios } \\
\text { (anuros) e } \\
\text { pequenos } \\
\text { mamíferos }\end{array}$ & $\begin{array}{l}\text { Estabelecer rede de fragmentos } \\
\text { florestais (reservas) que seja } \\
\text { mais eficiente para minimizar } \\
\text { ou interromper o processo de } \\
\text { perda de espécies, assim como } \\
\text { estabelecer áreas prioritárias para } \\
\text { ações de restauração }\end{array}$ & $\begin{array}{l}\text { Fragmentos } \\
\text { florestais (de } 2 \\
\text { a } 275 \text { ha) }\end{array}$ & $\begin{array}{l}\text { Área de } 100 \\
\mathrm{~km}^{2} \text { no estado } \\
\text { de São Paulo }\end{array}$ & $\begin{array}{l}\text { Simulated } \\
\text { annealing usando } \\
\text { complementaridade }\end{array}$ & MARXAN \\
\hline $\begin{array}{l}\text { Loyola et al. } \\
2013\end{array}$ & Brasil & Anfíbios & $\begin{array}{l}\text { Priorizar áreas para conservação } \\
\text { de espécies de anfíbios no } \\
\text { presente e em } 2050 \text { em cenário } \\
\text { de mudanças climáticas }\end{array}$ & $\begin{array}{l}\text { Células de } \\
0.5^{\circ}(\sim 50 \mathrm{x} \\
50 \mathrm{~km})\end{array}$ & $\begin{array}{l}\text { Mata Atlântica } \\
\text { Brasileira }\end{array}$ & $\begin{array}{l}\text { Simulated } \\
\text { annealing usando } \\
\text { complementaridade }\end{array}$ & MARXAN \\
\hline
\end{tabular}




\begin{tabular}{|c|c|c|c|c|c|c|c|}
\hline Referência & $\begin{array}{l}\text { País dos } \\
\text { Autores }\end{array}$ & $\begin{array}{c}\text { Dados } \\
\text { (Indicadores) } \\
\text { Utilizados* }\end{array}$ & Objetivos & $\begin{array}{l}\text { Resolução } \\
\text { Espacial } \\
\text { (grão)** }\end{array}$ & $\begin{array}{l}\text { Extensão } \\
\text { Espacial }\end{array}$ & Método de Seleção & Programa \\
\hline $\begin{array}{l}\text { Campos } \\
\text { et al. } 2014\end{array}$ & $\begin{array}{l}\text { Brasil e } \\
\text { Espanha }\end{array}$ & Anfibios & $\begin{array}{l}\text { Avaliar o desempenho } e \\
\text { a eficiência de grupos de } \\
\text { indicadores para representar a } \\
\text { diversidade de anfíibios }\end{array}$ & $\begin{array}{l}\text { Células de } \\
0.5^{\circ}(\sim 50 \mathrm{x} \\
50 \mathrm{~km})\end{array}$ & $\begin{array}{l}\text { Mata Atlântica } \\
\text { Brasileira }\end{array}$ & $\begin{array}{l}\text { Simulated } \\
\text { annealing usando } \\
\text { complementaridade }\end{array}$ & MARXAN \\
\hline $\begin{array}{l}\text { Crouzeilles } \\
\text { et al. } 2015\end{array}$ & $\begin{array}{l}\text { Brasil, } \\
\text { Inglaterra e } \\
\text { Austrália }\end{array}$ & Mamíferos & $\begin{array}{l}\text { Priorizar áreas para restauração } \\
\text { florestal incorporando a } \\
\text { disponibilidade de habitat para } \\
\text { duas espécies de mamíferos }\end{array}$ & $\begin{array}{l}\text { Paisagens de } \\
2000 \text { ha }\end{array}$ & $\begin{array}{l}\text { Mata Atlântica } \\
\text { Brasileira }\end{array}$ & $\begin{array}{l}\text { Simulated } \\
\text { annealing usando } \\
\text { complementaridade }\end{array}$ & MARXAN \\
\hline $\begin{array}{l}\text { Zwiener } \\
\text { et al. } 2017\end{array}$ & $\begin{array}{l}\text { EUA e } \\
\text { Brasil }\end{array}$ & $\begin{array}{l}\text { Plantas } \\
\text { lenhosas }\end{array}$ & $\begin{array}{l}\text { Propor e comparar áreas } \\
\text { prioritárias para conservação de } \\
\text { espécies de plantas lenhosas no } \\
\text { presente e em diferentes cenários } \\
\text { de mudanças climáticas e de uso } \\
\text { da terra em } 2050 \text { e } 2070\end{array}$ & $\begin{array}{l}\text { Células de } 5 \\
(\sim 10 \times 10 \\
\mathrm{km})\end{array}$ & $\begin{array}{l}\text { Mata Atlântica } \\
\text { Brasileira }\end{array}$ & $\begin{array}{l}\text { Priorização hierárquica } \\
\text { baseada em } \\
\text { complementaridade }\end{array}$ & ZONATION \\
\hline $\begin{array}{l}\text { de Lima \& } \\
\text { Pinto } 2018\end{array}$ & Brasil & Primatas & $\begin{array}{l}\text { Comparar diferentes tipos de } \\
\text { dados no estabelecimento } \\
\text { de áreas prioritárias para } \\
\text { conservação para } 11 \text { espécies de } \\
\text { primatas }\end{array}$ & $\begin{array}{l}\text { 1) Células de } \\
1^{\circ}(\sim 111 \mathrm{x} \\
111 \mathrm{~km}) \\
\text { 2) Células de } \\
0.5^{\circ}(\sim 50 \mathrm{x} \\
50 \mathrm{~km}) \\
\text { 3) Células de } \\
0.25^{\circ}(\sim 25 \mathrm{x} \\
25 \mathrm{~km})\end{array}$ & $\begin{array}{l}\text { Mata Atlântica } \\
\text { Brasileira }\end{array}$ & $\begin{array}{l}\text { Simulated } \\
\text { annealing usando } \\
\text { complementaridade }\end{array}$ & MARXAN \\
\hline $\begin{array}{l}\text { Vale et al. } \\
2018\end{array}$ & Brasil & Aves & $\begin{array}{l}\text { Estabelecer a efetividade da } \\
\text { rede de áreas protegidas para a } \\
\text { conservação de espécies de aves } \\
\text { endêmicas no presente e em } \\
2050 \text { em cenário de mudanças } \\
\text { climáticas }\end{array}$ & Municípios & $\begin{array}{l}\text { Mata Atlântica } \\
\text { Brasileira }\end{array}$ & $\begin{array}{l}\text { Simulated } \\
\text { annealing usando } \\
\text { complementaridade }\end{array}$ & MARXAN \\
\hline $\begin{array}{l}\text { de Oliveira } \\
\text { et al. } 2018\end{array}$ & Brasil & $\begin{array}{l}\text { Árvores } \\
\text { frutíferas }\end{array}$ & $\begin{array}{l}\text { Priorizar áreas para conservação } \\
\text { de nove espécies de árvores } \\
\text { frutíferas no presente e em cenário } \\
\text { de mudanças climáticas futuras } \\
\text { (2080 - 2100) e considerando } \\
\text { o efeito da invasão da jaqueira } \\
\text { (Artocarpus heterophyllus) }\end{array}$ & $\begin{array}{l}\text { Células de } \\
0.5^{\circ}(\sim 50 \mathrm{x} \\
50 \mathrm{~km})\end{array}$ & $\begin{array}{l}\text { Mata Atlântica } \\
\text { Brasileira }\end{array}$ & $\begin{array}{l}\text { Simulated } \\
\text { annealing usando } \\
\text { complementaridade }\end{array}$ & SITES*** \\
\hline $\begin{array}{l}\text { Larossa } \\
\text { et al. } 2019\end{array}$ & $\begin{array}{l}\text { Singapura, } \\
\text { Inglaterra e } \\
\text { Brasil }\end{array}$ & $\begin{array}{l}\text { Cobertura e } \\
\text { conectividade } \\
\text { florestal }\end{array}$ & $\begin{array}{l}\text { Priorizar a reserva de áreas em } \\
\text { terras agrícolas suficientes para } \\
\text { atender a uma meta ambiental de } \\
\text { restauração florestal e incremento } \\
\text { de conectividade, ao menor } \\
\text { custo possível, considerando o } \\
\text { pagamento de incentivos }\end{array}$ & Municípios & $\begin{array}{l}\text { Mata Atlântica } \\
\text { Brasileira }\end{array}$ & $\begin{array}{l}\text { Planejamento } \\
\text { dinâmico de } \\
\text { conservação espacial } \\
\text { considerando } \\
\text { simultaneamente } \\
\text { feedbacks ambientais } \\
\text { (conectividade } \\
\text { florestal) e sociais } \\
\text { (pagamento de } \\
\text { incentivos) em } \\
\text { áreas agrícolas, } \\
\text { implementado } \\
\text { com simulated } \\
\text { annealing usando } \\
\text { complementaridade }\end{array}$ & MARXAN \\
\hline
\end{tabular}

* Essa informação encontra-se no menor nível de resolução taxonômica que pôde ser identificado no artigo

** O grão é a unidade de análise

**** Interface do MARXAN para Arc View

Observando-se a utilização de informações sobre biodiversidade no conjunto dos 14 artigos que realizaram a PSC na $\mathrm{MAB}$, constatamos que os dados referentes às informações sobre espécies foram os mais usados nesses estudos levantados $(n=12)$. Apenas um trabalho utilizou dados de tipos de vegetação como informação de biodiversidade (Grelle et al. 2010) e outro usou dados de cobertura e conectividade florestal (Larrosa et al. 2018). Todos os demais trabalhos 
usaram informações relativas a espécies $(n=12)$, sendo que a maioria utilizou dados de vertebrados (Tabela 3). Dez estudos utilizaram um único grupo taxonômico, ressalvando-se que os mamíferos (especificamente, os primatas) foram utilizados em três dos estudos (Pinto \& Grelle 2009, 2011; de Lima \& Pinto 2018) e duas espécies de mamíferos foram utilizadas por Crouzeilles e colaboradores (2015). Dois estudos enfocaram as aves como grupo de análise (Loiselle et al. 2003, Vale et al. 2018) e três estudos os anfíbios (Becker et al. 2010, Loyola et al. 2013, Campos et al. 2014). Apenas um estudo tratou de mais de um grupo taxonômico (Felinks et al. 2011), analisando a dinâmica da composição de espécies de anfíbios e de pequenos mamíferos. Dois estudos analisaram plantas, sendo que Zwiener e colaboradores (2017) utilizaram dados sobre espécies de plantas lenhosas, e de Oliveira e colaboradores (2018) usaram dados de árvores frutíferas (Tabela 3).

Em relação às abordagens de análise, todos os estudos realizaram o processo de priorização de áreas por meio de algoritmos sequenciais baseados em complementaridade, 12 dos quais usaram o algoritmo simulated annealing (Tabela 3). Um dos estudos foi realizado no programa WORLDMAP (Loiselle et al. 2003), outro no ZONATION (Zwiener et al. 2017), enquanto os demais estudos utilizaram o instrumento computacional para realização da seleção de áreas denominado MARXAN $(\mathrm{n}=9)$ ou em uma variação sua denominada SITES $(n=3)$ (Tabela 3).

\section{Estudos não acadêmicos}

Dois estudos aplicaram métodos de priorização espacial vinculados à conservação da biodiversidade e às unidades de conservação: i) estudo oriundo da organização não governamental WWF (World Wildlife Fund) Brasil intitulado: "Visão da Biodiversidade da Biorregião Serra do Mar" (Scaramuza 2006); e ii) estudo conduzido pelo MMA, órgão público com competência para elaborar estratégias para a conservação da biodiversidade em âmbito nacional, denominado: "Áreas Prioritárias para a Conservação da Biodiversidade" (Brasil - MMA 2007a). O detalhamento desses estudos é apresentado na Tabela 4. As "Áreas Prioritárias para a Conservação da Biodiversidade" foram atualizadas em 2018 através da Portaria MMA n ${ }^{\circ} 463$, de 18 de dezembro de 2018, e a versão atualizada está disponível no site do MMA.
O estudo "Áreas Prioritárias para a Conservação da Biodiversidade" tem abrangência nacional, mas o processo de seleção das áreas prioritárias para conservação foi dividido por biomas. A presente análise restringiu-se, portanto, à parte do estudo que estabeleceu as áreas prioritárias para a $\mathrm{MAB}$, considerando os limites do bioma conforme estabelecido pelo Instituto Brasileiro de Geografia e Estatística (IBGE) (2004a, 2004b) e os encraves de MAB que ocorrem em outros biomas.

Observando-se a utilização de informações sobre a biodiversidade, é constatado que os dois estudos se valeram tanto de indicadores verdadeiros (true surrogates), que são os dados das espécies, quanto de indicadores estimadores (estimator surrogates) (ver Margules \& Sarkar 2007), que são os dados ambientais. O estudo do WWF empregou unidades fitogeomorfológicas (dado ambiental) e dados referentes a 120 espécies diferentes de animais e vegetais (dados de espécies), utilizando a modelagem preditiva de distribuição para as espécies e informações sobre distribuição das populações das espécies de anfíbios. O segundo estudo (o do MMA) usou unidades fitogeomorfológicas (dado ambiental) cuja metodologia de determinação difere do primeiro estudo, e utilizou dados de 409 espécies diferentes de animais (dados de espécies).

A maior parte das espécies abordadas nos dois trabalhos foi de animais, entretanto, o primeiro estudo lançou mão de nove espécies de vegetais, enquanto o segundo não utilizou espécies vegetais. Essa utilização preponderante de espécies animais se concentrou em espécies de vertebrados - 109 no estudo da WWF e 311 no estudo do MMA. Neste último, foram utilizadas 98 espécies de invertebrados, e no da WWF não foram utilizadas espécies de invertebrados. Entre os vertebrados, as espécies de aves foram as mais utilizadas: 48 no estudo da WWF e 113 espécies no estudo do MMA.

Os dois estudos realizaram o processo de seleção de áreas prioritárias para conservação por meio de instrumentos computacionais MARXAN e CPLAN (Tabela 4). Esses estudos são apresentados em um formato de publicação com muito mais espaço para o detalhamento das etapas e da sua realização, além de se reportarem à ocorrência de oficinas e reuniões com o intuito de fundamentar a elaboração e a construção das recomendações para sua aplicação, o que não se observa nos 
estudos acadêmicos. O segundo estudo gerou uma portaria que subsidia legalmente as políticas públicas $e$ a legislação que regem as ações de conservação do Governo Brasileiro em todo o território nacional.

A elaboração do projeto "Visão da Conservação de Biodiversidade da Ecorregião Serra do Mar" se deu no âmbito do Programa para Conservação da Biodiversidade nos Sítios do Patrimônio Mundial Natural do Brasil, que tem como objetivo consolidar e promover a gestão integrada nos sítios nacionais. Sua finalidade é servir como base de planejamento para as ações de conservação do WWF-Brasil nesse território.

A elaboração e a revisão das "Áreas Prioritárias para Conservação da Biodiversidade" foi um dos desdobramentos da participação do Brasil na Convenção sobre Diversidade Biológica (CDB), assinada em 1992. Essa convenção definiu como uma das principais metas para os países signatários o estabelecimento e o manejo adequado de um sistema de áreas protegidas. $\mathrm{O}$ Ministério do Meio Ambiente realizou, entre 1998 e 2000, a primeira "Avaliação e Identificação das Áreas e Ações Prioritárias para a Conservação dos Biomas Brasileiros". Desse modo, a indicação de sistemas de áreas prioritárias para a conservação estendeu-se para todo o país no final da década de 90. A atualização das áreas prioritárias para conservação foi feita em 2007, a partir da utilização do protocolo de métodos do PSC (Brasil - MMA 2007a).

Tabela 4 - Estudos não acadêmicos que aplicaram Planejamento Sistemático para Conservação na Mata Atlântica Brasileira.

\begin{tabular}{|c|c|c|c|c|c|c|c|}
\hline Referência & Organização & $\begin{array}{l}\text { País dos } \\
\text { Autores }\end{array}$ & $\begin{array}{c}\text { Dados (Indicadores) } \\
\text { Utilizados* }\end{array}$ & 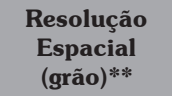 & $\begin{array}{l}\text { Extensão } \\
\text { Espacial }\end{array}$ & $\begin{array}{l}\text { Método de } \\
\text { Seleção }\end{array}$ & Programa \\
\hline $\begin{array}{l}\text { Scaramuzza } \\
\text { et al. } 2011\end{array}$ & $\begin{array}{l}\text { Organização não } \\
\text { governamental: } \\
\text { WWF-Brasil }\end{array}$ & Brasil & $\begin{array}{l}\text { Unidades fitogeomorfológicas, } \\
\text { espécies de palmeiras, anfíbios, } \\
\text { aves e mamíferos }\end{array}$ & $\begin{array}{l}\text { Hexágonos de } \\
5000 \text { ha }\end{array}$ & $\begin{array}{l}\text { Ecorregião da } \\
\text { Serra do Mar (ES, } \\
\text { MG, RJ, SP, PR, } \\
\text { SC, RS) }\end{array}$ & $\begin{array}{l}\text { Simulated } \\
\text { annealing usando } \\
\text { complementaridade }\end{array}$ & $\begin{array}{l}\text { MARXAN / } \\
\text { CPLAN }\end{array}$ \\
\hline Brasil 2007 & $\begin{array}{l}\text { Organização } \\
\text { governamental: } \\
\text { Ministério do Meio } \\
\text { Ambiente }\end{array}$ & Brasil & $\begin{array}{l}\text { Unidades fitogeomorfológicas, } \\
\text { espécies de invertebrados, } \\
\text { peixes, anfíbios, répteis, aves e } \\
\text { mamíferos }\end{array}$ & $\begin{array}{l}\text { Hexágonos de } \\
6000 \text { ha }\end{array}$ & $\begin{array}{l}\text { Mata Atlântica } \\
\text { Brasileira (limite } \\
\text { do bioma, IBGE } \\
\text { 2004) }\end{array}$ & $\begin{array}{l}\text { Simulated } \\
\text { annealing usando } \\
\text { complementaridade }\end{array}$ & $\begin{array}{l}\text { MARXAN / } \\
\text { CPLAN }\end{array}$ \\
\hline
\end{tabular}

* Essa informação encontra-se no menor nível de resolução taxonômica que pôde ser identificado no artigo

** O grão é a unidade de análise

\section{Discussão}

No panorama traçado neste trabalho sobre as aplicações de Planejamento Sistemático de Conservação na $\mathrm{MAB}$, foi possível identificar que a maioria dos estudos representa aplicações metodológicas da etapa de priorização dentro do framework do PSC: realizadas no ambiente terrestre; em escala regional; utilizando dados de espécies, sobretudo de animaisvertebrados; através de métodos de análise de complementaridade e insubstituibilidade, implementadas principalmente no programa MARXAN. Esse perfil tem várias características comuns já apontadas na literatura sobre planejamento sistemático de conservação (e.g. Pinto \& Grelle 2009a, McIntosh et al. 2018, Ribeiro \& Ateu 2019).
A biologia da conservação é considerada principalmente do ponto de vista macroscópico de grandes organismos, como aves e mamíferos, que são predominantemente estudados nos artigos científicos dessa disciplina (Clark \& May 2002). Nesse contexto, os diferentes grupos de organismos atraem o interesse humano de forma distinta por diversas razões, sendo que na biologia da conservação o apelo às espécies mais carismáticas como alvo de conservação advém da necessidade de apoio público. Nos estudos aqui levantados, se observa uma tendência para a maior utilização de dados sobre vertebrados terrestres. A preferência pelo uso de informações sobre espécies ou grupos taxonômicos específicos parece ser uma tendência dos estudos de PSC (Ribeiro \& Ateu 2019). 
Uma das análises que podem ser feitas no âmbito do PSC é o reconhecimento do quanto os alvos para a conservação vêm sendo alcançados nos sistemas de áreas protegidas estabelecidas (Margules \& Pressey 2000). Essa tarefa é conhecida como análise de lacunas para a conservação $e$ pode ser considerada como a pesquisa que verifica a eficiência dos sistemas de áreas protegidas existentes em representar a biodiversidade (Pinto \& Grelle 2009b). A análise de lacunas para a conservação pode ser realizada na etapa de revisão do sistema de áreas para conservação anterior à etapa de priorização de novas áreas com potencial para ações de conservação, quando são utilizados algoritmos de priorização baseados na complementaridade (Margules \& Sarkar 2007).

Três estudos revisados aqui realizaram esse tipo de análise como uma etapa preliminar à seleção de cenário de sistemas de áreas de conservação, avaliando a representatividade do sistema de unidades de conservação da MAB (Pinto \& Grelle 2009b, Grelle et al. 2010, Scaramuzza et al. 2011). Grelle e colaboradores (2010), por meio da análise de lacunas para a conservação, determinaram que, no sistema de unidades de conservação de proteção integral do estado do Rio de Janeiro existente, estão representados 15 dos 19 tipos de vegetação que ocorrem no estado. Pinto \& Grelle (2009b), por meio da análise de lacunas para a conservação, determinaram que, no sistema de unidades de conservação da $\mathrm{MAB}$ existente, estão representadas $89 \%$ das espécies endêmicas de primatas. Assim, esse sistema pode ser considerado razoavelmente representativo para a biodiversidade de primatas, uma vez que não foi desenhado com esse objetivo. Scaramuzza e colaboradores (2011) realizaram uma análise de lacunas e identificaram o nível de proteção de 202 objetos de conservação conferidos pelo sistema de unidades de conservação de proteção integral da MAB da Ecorregião da Serra do Mar. No caso, os 202 objetos de conservação foram avaliados segundo três níveis: desprotegido (sem representatividade alguma em UCs), com proteção parcial (com alguma proteção em UCs, mas ainda sem atingir a meta) e protegido (com a meta de proteção atingida). O nível de proteção atingido pelos objetos de conservação é de $51 \%$ (104 objetos). Aquém das metas, estão 74 objetos (37\%) e, completamente desprotegidas, 24 (12\%). Assim, $84 \%$ dos objetos de conservação estão representados no sistema de unidades de conservação de proteção integral da $\mathrm{MAB}$ restrita à Ecorregião da Serra do Mar.

Esse conjunto de unidades de conservação foi o primeiro a ser estabelecido no Brasil (Diegues 2001, Cabral 2002, Ferreira 2004, Medeiros 2006) e, portanto, a maior parte das suas unidades de conservação foi criada por outros critérios que não a representatividade de objetivos de conservação num contexto tradicional de seleção de áreas para conservação, ou seja, sem uma definição a priori de objetivos explícitos (Pressey et al. 1993, Pressey 1994). Entretanto, os três estudos indicam que, mesmo que criado sem essa intenção a priori, associado ao sistema de unidades de conservação da MAB existente nele ou em parte dele, observase uma contribuição significativa para conservação de alvos explícitos para a conservação da biodiversidade.

Paglia (2013) levantou 20 iniciativas de priorização de áreas para conservação da biodiversidade na MA, entre 1993 e 2011, que utilizaram diversos métodos além do PSC. Das 20 iniciativas, apenas quatro utilizaram o PSC. Citamos nesta revisão dois deles (Brasil - MMA 2007a e Scaramuzza et al. 2011). Os outros dois apontados por Paglia (2013) consistem em uma comunicação em congresso (Paglia et al. 2004), e o outro trata de um artigo da revista Megadiversidade, que focou na identificação de áreas insubstituíveis para a conservação da biodiversidade na Cadeia do Espinhaço, estados de Minas Gerais e Bahia, incluindo pequenas porções da MAB e, principalmente, áreas do cerrado.

Paglia (2013) também observou que a utilização dos estudos na elaboração de políticas públicas é considerada como medida de aplicação desses estudos em ações efetivas de conservação. Assim, das 20 iniciativas levantadas por esse autor, apenas metade gerou políticas públicas $e$ ações efetivas de conservação. $\mathrm{Na}$ presente revisão, em apenas dois estudos (Brasil - MMA 2007a e Scaramuzza et al. 2011) observamos a reversão dos resultados em políticas públicas, o que demonstra a baixa reversão dos estudos em ações efetivas de conservação - fato observado também em os outros estudos de PSC, conforme Knight et al. (2009).

Os métodos de priorização espacial para a seleção de áreas podem, ainda, tratar de outras estratégias para a conservação na $\mathrm{MAB}$, como a restauração de áreas, o estabelecimento de 
corredores e a formação de mosaicos, como de fato se observa no estabelecimento das Áreas Prioritárias para Conservação da Biodiversidade do MMA (Brasil - MMA 2007a). A identificação de áreas para restauração também é feita em quatro estudos levantados (Felinks et al. 2011, Crouzeilles et al. 2015, Zwiener et al. 2017, Larossa et al. 2019). Esses últimos três estudos estão sob a influência do Pacto pela Restauração da Mata Atlântica, iniciado em 2009 (Pinto et al. 2014).

Os recursos disponíveis para a conservação são limitados, sendo necessária a adoção de estratégias planejadas para sua alocação. Esse fato levou ao surgimento da ciência do PSC (Margules \& Pressey 2000). Assim, apesar do grande apelo metodológico do PSC, a maioria dos estudos na MAB não teve seus resultados revertidos em políticas públicas para a conservação da biodiversidade. Por outro lado, ainda que em pequeno número, foi positiva a constatação que um órgão público (MMA) e uma grande ONG de conservação (WWF) tenham utilizado o PSC para subsidiar políticas públicas e projetos de conservação.

\section{Agradecimentos}

Os autores gostariam de agradecer aos revisores da Revista Biodiversidade Brasileira, pelas importantes contribuições dadas ao artigo. $\mathrm{RD}$ gostaria de agradecer aos colegas do IBAMA e ICMBio, em especial à chefia da Coordenação de Criação de Unidades de Conservação (COCUC) e às demais instâncias da DIMAN, da DIBIO, e ao Conselho Gestor do ICMBio. Os autores agradecem também ao Programa de Pós-Graduação Profissional Biodiversidade em Unidades de Conservação, da Escola Nacional de Botânica Tropical/Jardim Botânico do Rio de Janeiro, por todo o suporte prestado ao longo do desenvolvimento deste trabalho. Ao senhor Sashi Jaiswal, pela correção do resumo em inglês e a tradução do resumo em espanhol do presente artigo.

\section{Referências Bibliográficas}

Araújo MAR. 2012. A Seleção e o Desenho de Unidades de Conservação. In: Orgs. Nexucs. Unidades de Conservação no Brasil - O Caminho da Gestão para Resultados. São Carlos. Ed. Rima. 536p.
Ayres JM et al. 2005. Os corredores ecológicos das florestas tropicais do Brasil. Sociedade Civil Maminaurá. 256p.

Becker CG, Loyola RD, Haddad CFB \& Zamudio KR. 2010. Integrating species life-history traits and patterns of deforestation in amphibian conservation planning. Diversity and Distributions. DOI: 10.1111/j.14724642.2009.00625.x.

Brasil. 2000. Lei $\mathrm{n}^{\circ}$ 9.605, de 18 de julho 2000. Disponível em: <https://www.planalto.gov.br/ccivil_03/ leis/15197.htm > . Acesso em: 20/11/2013.

Brasil. Ministério do Meio Ambiente. 2007a. Atualização: Portaria MMA $n^{\circ}$ 9, de 23 de janeiro de 2007. Disponível em: <http://www.mma.gov.br/publicacoes/ biodiversidade/category/142-serie-biodiversidade $>$. Acesso em: 15/12/2013.

Brasil. 2007b. MMA - IBAMA. Informe nacional sobre áreas protegidas no Brasil/Ministério do Meio Ambiente, Secretaria de Biodiversidade e Florestas, Departamento de Áreas Protegidas. 124p.

Cabral NRAJ \& Souza MP. 2002. Área de Proteção Ambiental: planejamento e gestão de paisagens protegidas. Rima. 154p.

Campos FS, Trindade-Filho J, Brito D, Lorente GA \& Mirco S. 2014. The effciency of indicator groups for the conservation of amphibians in the Brazilian Atlantic Forest. Ecology and Evolution, 4(12): 2505-2514, 2014. Doi:10.1002/ece3.1073.

CEPF. Critical Ecosystem Partnership Fund. 2001. Ecosystem profile: Atlantic Forest biodiversity hotspot. Brazil. Final version. December 11, 2001. Critical Ecosystem Partnership Fund (CEPF), Conservation International. Disponível em: <http://www.cepf.net/ Documents/final.atlanticforest.ep.pdf $>$. Acesso em: 13/09/2012.

Clark JA \& May RM. 2002. Taxonomic bias in conservation research. Science, 297(5579): 191-192.

Crouzeilles R, Beyer HL, Mills M, Grelle EV \& Possingham HP. 2015. Incorporating habitat availability into systematic planning for restoration: a speciesspecifc approach for Atlantic Forest mammals. Diversity and Distributions, 1-11. DOI: $10.1111 / \mathrm{j} .1472-$ 4642.2009.00625.x.

de Lima AA \& Pinto MP. 2018. Influence of extent and data type on spatial conservation prioritization. Ecoinf (2018). Doi:10.1016/ j.ecoinf.2018.07.003.

de Marques AAB, Schneider M \& Peres CA. 2016. Human population and socioeconomic modulators of conservation performance in 788 Amazonian and Atlantic Forest reserves. PeerJ4:e2206https://doi. org/10.7717/peerj.2206. 
de Oliveira G, de Souza Barreto B, da Silva dos Santos D, Queiroz de Matos V \& Seara Santos MC. 2018. Combining the effects of biological invasion and climate change into systematic conservation planning for the Atlantic Forest. Biol Invasions, 20: 2753-2765. DOI: https://doi.org/10.1007/s10530-018-1727-y

Diegues AC. 2001. O mito moderno da natureza intocada. Hucitec. 161p.

Felinks B, Pardini R, Dixo M, Follner K, Metzger JP \& Henle K. 2011. Effects of species turnover on reserve site selection in a fragmented landscape. Biodiversity. Conservation, 20: 1057-1072. DOI: 10.1007/s10531011-0015-2.

Ferreira IV. Uma política nacional para as áreas protegidas brasileiras. Anais do IV Congresso Brasileiro de Unidades de Conservação. Curitiba, Fundação O Boticário de Proteção à Natureza \& Rede Pró Unidades de Conservação, 2: 172-176, 2004.

Forzza RC et al. New Brazilian Floristic List Highlights Conservation Challenges, BioScience, 62(1): 1, 39-45, 2012. DOI:https://doi.org/10.1525/bio.2012.62.1.8.

Grelle CEV, Lorini ML \& Pinto MP. Reserve selection based on vegetation in the Brazilian Atlantic Forest. Natureza a Conservação, 8(1): 46-53, 2010.

Hopkinson P, Evans J \& Gregory RD. National-scale conservation assessments atan appropriate resolution. Diversity and Distributions, 6: 195-204, 2000.

Iacona GD, Possingham HP \& Bodee M. 2017. Waiting can be an optimal conservation strategy, even in a crisis discipline. www.pnas.org/cgi/doi/10.1073/ pnas. 1702111114.

IBGE - Instituto Brasileiro de Geografia e Estatística. 2004a. Mapa de Biomas do Brasil. Disponível em: www.ibge.gov.br/home/geociencias/default_prod. shtm\#USO. Acesso em: 15/12/2012.

IBGE - Instituto Brasileiro de Geografia e Estatística. 2004b. Nota Técnica do Mapa de Biomas do Brasil. Disponívelem:http://www.ibge.gov.br/home/geociencias/ default_prod.shtm\#MAPAS. Acesso em: 15/12/2002.

IUCN. 1994. World Conservation Union. Guidelines for Protected Area Management Categories IUCN, Gland, Switzerland, and Cambridge.

Izquierdo AE \& Clark ML. Spatial analysis of conservation priorities based on ecosystem services in the Atlantic forest region of Misiones, Argentina. Forests, 3: 764-786, 2012.

Kalamandeen M \& Gillson L. Demything "wilderness": implications for protected area designation and management. Biodiversity Conservation, 16: 165-182, 2007. DOI: 10.1007/s10531-006-9122-x.
Knight AT, Cowling RM, Possinghami HP \& Wilson KA. 2009. From theory to practice: designing and situating spatial prioritization approaches to better implement conservation action, p. 249-259. In: Moilanen A, Wilson KA \& Possingham HP (eds.). Spatial conservation prioritization quantitative methods and computational tools, 1st edition. Oxford University Press.

Larrosa C, Carrasco LR, Tambosi LR, Banks-Leite C \& Milner-Gulland EJ. Spatial conservation planning with ecological and economic feedback effects. Biological Conservation 237: 308-316, 2019. DOI: https://doi. org/10.1016/j.biocon.2019.06.015.

Laurance WF. Conserving the hottest of the hotspots. Biological Conservation, 142: 1137, 2009.

Lemes P \& Loyola RD. Accommodating Species Climate-Forced Dispersal and Uncertainties in Spatial Conservation Planning. PLoS ONE 8(1): e54323, 2013. DOI: 10.1371/journal.pone.0054323.

Loiselle BA et al. Avoiding Pitfalls of Using Species Distribution Models in Conservation Planning. Conservation Biology, 17(6): 1591-1600, 2003.

Loyola $\mathrm{RD}$ et al. A straightforward conceptual approach for evaluating spatial conservation priorities under climate change. Biodiversity and Conservation, 22(2): 483-495, 2013. DOI:10.1007/s10531-0120424-x.

Margules CR \& Pressey RL. Systematic conservation planning. Nature, 405: 243-253, 2000.

Margules C \& Sarkar S. 2007. Systematic conservation planning. Cambridge, Cambridge University Press. 270p.

Marinoni L \& Peixoto AL. As coleções biológicas como fonte dinâmica e permanente de conhecimento sobre a biodiversidade. Cienc. Cult., v. 62, n. 3, 2010. Disponível em: http://cienciaecultura.bvs. br/scielo.php?script = sci_arttext\&pid $=$ S0009. $67252010000300021 \& \operatorname{lng}=e^{-}$\& $\mathrm{nrm}=$ iso $>$. Acesso em: 13/2/2014.

McIntosh EJ, Chapman S, Kearney SG et al. Absence of evidence for the conservation outcomes of systematic conservation planning around the globe: a systematic map. Environ. Evid. 7: 22, 2018.

Medeiros R. Evolução das tipologias e categorias de áreas protegidas no Brasil. Campinas, Ambient. Soc. v. 9, n. 1, 2006.

Mittermeier R et al. 2005. Hotspots Revisited: Earth's Biologically Richest and Most Endangered Terrestrial Ecoregions an Inspiring Update to an Unparalleled Biodiversity Resource. Cemex Conservation Book Series 12. Conservation International. 391p. 
Moilanen A, Wilson KA \& Possingham HP. 2009 Spacial Conservation Priorization - Quantitative Methods \& computational tools Oxford: Oxford University Press.

Morsello C. 2001. Áreas protegidas públicas e privadas: seleção e manejo. Annablume, FAPESPE. 344p.

Myers N, Mittermeier RA, Mittermeier CG, da Fonseca GAB \& Kent J. 2000. Biodiversity hotspots for conservation priorities. Nature 403, 853-858. DOI: 10.1038/35002501.

Oliveira-Filho AT \& Fontes MAL. Patterns of Floristic Differentiation among Atlantic Forests in Southeastern Brazil and the Influence of Climate. Biotropica, 32(4b): 793-810, 2000.

Ovando XMC, Miranda MJ, Loyola R \& Cuezzo MG. Identifying priority areas for invertebrate conservation using land snails as models. Journal for Nature Conservation, 50: 125707, 2019. DOI: https://doi. org/10.1016/j.jnc.2019.04.004.

Paglia AP. 2013. Avaliação das abordagens e iniciativas de priorização de ações para a conservação da biodiversidade na Mata Atlântica, p. 88-134. In: Mapeamentos para a conservação e recuperação da biodiversidade na Mata Atlântica: em busca de uma estratégia espacial integradora para orientar ações aplicadas. Série Biodiversidade 49 - MMA.

Paglia AP \& Pinto LP. 2010. Biodiversidade da Mata Atlântica, p. 102-118. In: Marone E, Riet D \& Melo T. (orgs.). Brasil Atlântico - um país com a raiz na mata. Instituto BioAtlântica.

Paglia AP et al. 2004. Lacunas de conservação e áreas insubstituíveis para vertebrados ameaçados da Mata Atlântica, p. 39-50. In: Anais do IV Congresso Brasileiro de Unidades de Conservação. Volume II.

Pinto MP \& Bini LM. Vieses geográficos e taxonômicos nas pesquisas sobre seleção de reservas: uma análise quantitativa de 1992 a 2004. Natureza \& Conservação, 6(1): 35-45, 2008.

Pinto MP \& Grelle CEV. Seleção de reservas: Estudos na América do Sul e revisão de conceitos, Oecologia Brasiliensis 13(3): 498-517, 2009a.

Pinto MP \& Grelle CEV. Reserve selection and persistence: Complementing the existing atlantic forest reserve system. Biodiversity and Conservation, 18(4): 957-968, $2009 \mathrm{~b}$.

Pinto MP \& Grelle CEV. Minimizing conservation conflict for endemic primate species in Atlantic forest and uncovering knowledge bias. Environmental Conservation, 39(1): 30-37, 2011.

Pinto SR et al. Governing and Delivering a BiomeWide Restoration Initiative: The Case of Atlantic Forest Restoration Pact in Brazil. Forests, 2014, 5: 2212-2229. DOI: $10.3390 / f 5092212$.
Prendergast JR, Quinn RM \& Lawton JH. The gaps between theory and practice in selecting nature reserves. Conservation Biology 13: 484-492, 1999.

Pressey RL. Ad hoc reservations: foward or backward steps in developing representative reserves systems? Conservation Biology 8: 662-668, 1994. DOI: 10.1046/ j.1523-1739.1994.08030662.x.

Pressey RL, Humphries CJ, Margules CR, Vane-Wright RI \& Williams PH. Beyond opportunism: key principles for systematic reserve selection. Trends in Ecology and Evolution 8: 124-128, 1993. DOI:10.1016/01695347(93)90023-I.

Pullin AS \& Knight TM. Do conservation managers use scientific evidence to support their decision-making? Biological Conservation 19: 245-252, 2004.

Pyšek P, Richardson DM, Pergl J, Jarošík V, Sixto vá Z \& Weber E. Geographical and taxonomic biases in invasion ecology. Trends in Ecology and Evolution, 23(5): 237-244, 2008.

Ribeiro RB \& Ateu M. Systematic conservation planning: Trends and patterns among highly-cited papers. Journal for Nature Conservation 50, 125714, 2019.

Ribeiro MC, Metzger JP, Martensen AC, Ponzoni F \& Hirota MM. Brazilian Atlantic forest: how much is left and how is the remaining forest distributed? Implications for conservation. Biological Conservation, 142: 1141-1153, 2009.

Runte A. 1979. National Parks: The American experience. Lincoln and London: University of Nebraska Press. 240p.

Scaramuzza CAM et al. 2011. Visão de Biodiversidade da Ecorregião Serra do Mar. WWF-Brasil. Disponível em: http://www.wwf.org.br/?28724/Visao-da-Biodiversidadeda-Ecorregiao-Serra-do-Mar.

Silva JMC \& Casteleti CHM. 2003. Status of the biodiversity of the Atlantic Forest of Brazil, p. 43-59. In: Galindo-Leal C \& Câmara IG (eds.). The Atlantic Forest of South America: Biodiversity Status, Threats, and Outlook. Washington: Center for Applied Biodiversity Science CABS e Island Press.

Tabarelli M, Pinto LP, Silva JMC, Hirota MM \& Bedê LC. Desafios e oportunidades para a conservação da biodiversidade na Mata Atlântica brasileira. Megadiversidade. v. 1, n. 1, 2005.

Trindade-Filho J, de Carvalho RA, Brito D \& Loyola $\mathrm{RD}$. How does the inclusion of Data Deficient species change conservation priorities for amphibians in the Atlantic Forest? Biodiversity and Conservation, 21(10): 2709-2718, 2012.

Vasconcelos TS \& Prado VHM. Climate change and opposing spatial conservation priorities for anuran 
protection in the Brazilian hotspots. Journal for Nature Conservation, 49: 118-124, 2019. DOI: https://doi. org/10.1016/j.jnc.2019.04.003.

Vale MM, Souza TV, Alves MAS \& Crouzeilles R. 2018. Planning protected areas network that are relevant today and under future climate change is possible: the case of Atlantic Forest endemic birds. PeerJ6:e4689. DOI10.7717/peerj.4689.

Verbeek A, Debackere K, Luwel M \& Zimmermann $\mathrm{E}$. Measuring the progress and evolution in science and technology - I: The multiple uses of bibliometric indicators. International Journal of Management Reviews, 4(2): 179-211, 2-2, 2002.

Wilson JRU, Proches S, Braschler B, Dixon ES \& Richardson DM. The (bio)diversity of science reflects the interests of society. Frontiers in Ecologyandthe Environment, 5: 409-414, 2007.

Zwiener VP et al. Planning for conservation and restoration under climate and land use change in the Brazilian Atlantic Forest. Diversity and Distributions, 1-12, 2017. DOI: 10.1111/ddi.12588.

Biodiversidade Brasileira - BioBrasil.

Edição temática: Diálogos entre a Academia e a Gestão de Áreas Protegidas:

Programa de Pós-Graduação Profissional - Biodiversidade em Unidades de Conservação

$$
\text { n. 2, } 2020
$$

http://www.icmbio.gov.br/revistaeletronica/index.php/BioBR

Biodiversidade Brasileira é uma publicação eletrônica científica do Instituto Chico Mendes de

Conservação da Biodiversidade (ICMBio) que tem como objetivo fomentar a discussão e a disseminação de experiências em conservação e manejo, com foco em unidades de conservação $e$ espécies ameaçadas.

ISSN: 2236-2886 